Physics

Physics Research Publications

\title{
Tests of new physics from precise measurements of the Casimir pressure between two gold-coated plates
}
R. S. Decca
D. Lopez
E. Fischbach

G. L. Klimchitskaya

D. E. Krause

V. M. Mostepanenko 


\title{
Tests of new physics from precise measurements of the Casimir pressure between two gold-coated plates
}

\author{
R. S. Decca, ${ }^{1}$ D. López, ${ }^{2}$ E. Fischbach, ${ }^{3}$ G. L. Klimchitskaya, ${ }^{4}$ D. E. Krause, ${ }^{5,3}$ and V. M. Mostepanenko ${ }^{6}$ \\ ${ }^{1}$ Department of Physics, Indiana University-Purdue University Indianapolis, Indianapolis, Indiana 46202, USA \\ ${ }^{2}$ Bell Laboratories, Lucent Technologies, Murray Hill, New Jersey 07974, USA \\ ${ }^{3}$ Department of Physics, Purdue University, West Lafayette, Indiana 47907, USA \\ ${ }^{4}$ North-West Technical University, Millionnaya St. 5, St. Petersburg, 191065, Russia \\ ${ }^{5}$ Physics Department, Wabash College, Crawfordsville, Indiana 47933, USA \\ ${ }^{6}$ Noncommercial Partnership "Scientific Instruments," Tverskaya St. 11, Moscow, 103905, Russia \\ (Received 11 September 2006; published 23 April 2007)
}

\begin{abstract}
A micromechanical torsion oscillator has been used to strengthen the limits on new Yukawa forces by determining the Casimir pressure between two gold-coated plates. By significantly reducing the random errors and obtaining the electronic parameters of the gold coatings, we were able to conclusively exclude the predictions of large thermal effects below $1 \mu \mathrm{m}$ and strengthen the constraints on Yukawa corrections to Newtonian gravity in the interaction range from $29.5 \mathrm{~nm}$ to $86 \mathrm{~nm}$.
\end{abstract}

DOI: 10.1103/PhysRevD.75.077101

PACS numbers: $14.80 .-\mathrm{j}, 04.50 .+\mathrm{h}, 11.25 . \mathrm{Mj}, 12.20 . \mathrm{Fv}$

The Casimir effect [1] has attracted considerable attention recently because it is a direct manifestation of the existence of the quantum vacuum, and has far-reaching multidisciplinary consequences. According to Casimir's prediction there is a force acting between electrically neutral surfaces which arises from the modification of the zero-point oscillations due to the presence of material boundaries. (No such force exists in the framework of classical electrodynamics.) The Casimir effect finds applications in elementary particle physics (bag model of hadrons, compactification of extra dimensions), gravitation and cosmology (cosmological constant, dark matter) and in condensed matter physics (layered structures, wetting processes). It is currently being applied in nanotechnology, and to obtain constraints on new physics beyond the standard model (references on all of the above applications can be found in the monographs [2] and reviews [3-5]). During the last decade many experiments measuring the Casimir force between metals, and most recently between a metal and a semiconductor, have been performed using torsion pendulums, atomic force microscopes, and micromechanical torsional oscillators [6-11].

Here we report limits of new physics obtained from an experimental determination of the Casimir pressure between two Au-coated plates which incorporated three significant improvements over all previous measurements. First, a new experimental procedure was implemented which permitted us to repeat the measurements over a wide separation region many times in such a way that data were acquired at practically the same points for each repetition. Second, the random experimental error was substantially reduced compared to the systematic error, as required for precise measurements. The total experimental relative error determined at $95 \%$ confidence varies from $0.19 \%$ at the shortest separation of $z=160 \mathrm{~nm}$ to only $9.0 \%$ at the largest separation of $z=750 \mathrm{~nm}$. Third, the electronic parameters of Au films used for the extrapolation of the tabulated optical data to low frequencies (the plasma frequency $\omega_{p}$ and the relaxation parameter $\gamma$ ) were determined using the measured temperature dependence of the film's resistivity. All these improvements permitted us to obtain both precise and accurate experimental and theoretical results over a wide separation range. Our results lead to a more conclusive choice among the competing approaches to the thermal Casimir force (see, e.g., Refs. [12-15]), and to stronger constraints on models of non-Newtonian gravity.

In this experiment, the Casimir pressure between two Au-coated parallel plates was determined dynamically by means of a micromechanical torsional oscillator consisting of a plate suspended at two opposite points by serpentine springs, and a sphere above it attached to an optical fiber (all details of the experimental setup are presented in Ref. [11]). The separation between the sphere of radius $R=151.3 \pm 0.2 \mu \mathrm{m}$ and the plate was varied harmonically, $\tilde{z}(t)=z+A_{z} \cos \left(\omega_{r} t\right)$, where $\omega_{r}$ is the resonant angular frequency of the oscillator under the influence of the Casimir force from the sphere, and $A_{z} / z \ll 1$. (The values of $A_{z}$ at each $z$ were chosen such that the oscillator exhibited a linear response.) The resonant frequency $\omega_{r}$ is related to the natural angular frequency $\omega_{0}=2 \pi \times$ (713.25 \pm 0.02$) \mathrm{Hz}$ by [9-11]

$$
\omega_{r}^{2}=\omega_{0}^{2}\left[1-\frac{b^{2}}{I \omega_{0}^{2}} \frac{\partial F(z)}{\partial z}\right]
$$

where $b$ is the lever arm, $I$ is the moment of inertia, $b^{2} / I=$ $(1.2432 \pm 0.0005) \mu \mathrm{g}^{-1}$, and $F(z)$ is the Casimir force acting between the sphere and the plate. By measuring the frequency shift, we determine $\partial F / \partial z$ from Eq. (1), and using the proximity force approximation (PFA) arrive at the Casimir pressure between two parallel plates 

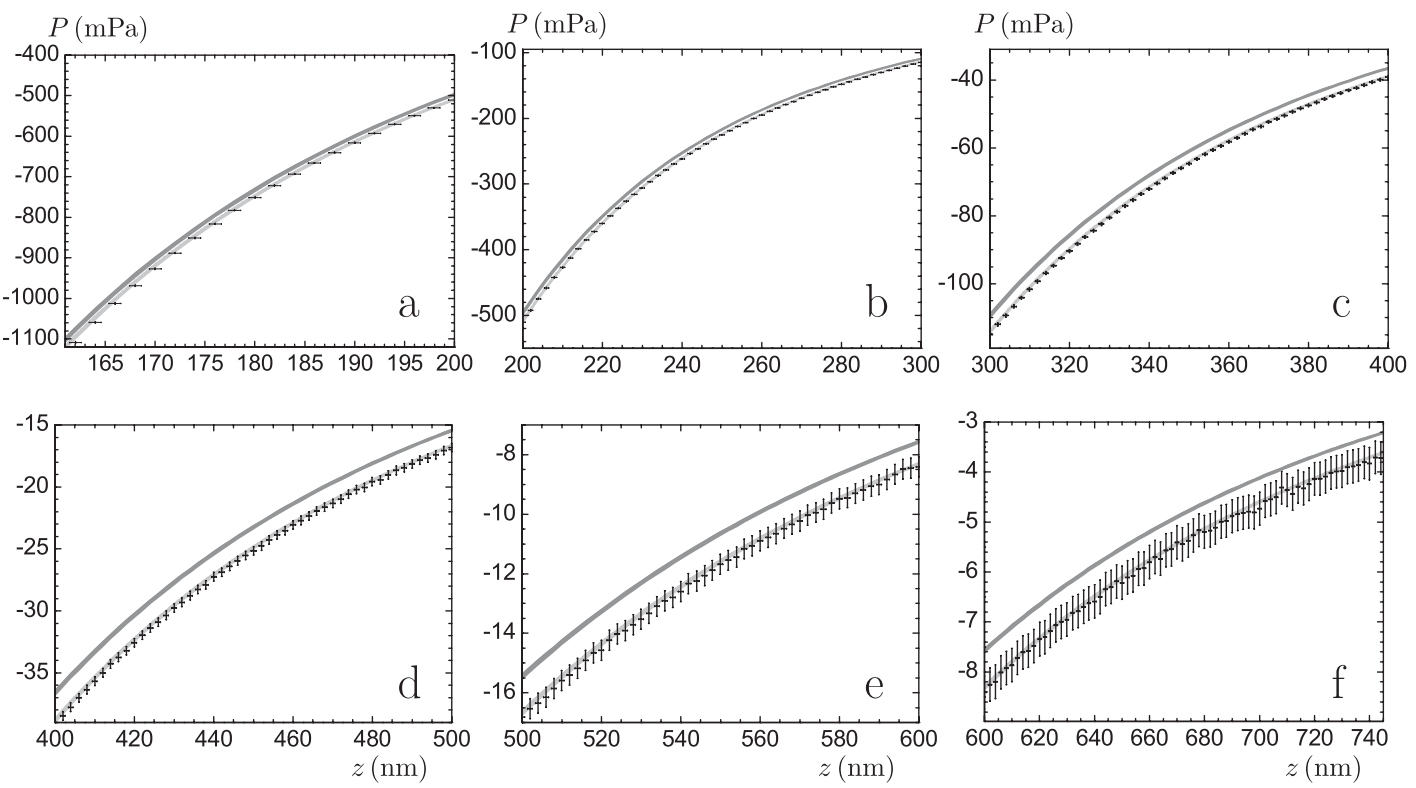

FIG. 1. Experimental data for the Casimir pressure as a function of separation $z$. Absolute errors are shown by black crosses in different separation regions (a-f). The light- and dark-gray bands represent the theoretical predictions of the impedance and Drude model approaches, respectively. The vertical width of the bands is equal to the theoretical error, and all crosses are shown in true scale.

$$
P(z)=-\frac{1}{2 \pi R} \frac{\partial F(z)}{\partial z}
$$

Although recent results [16] show that the error in using the PFA is less than $z / R$, we assume in our error analysis that the error is $z / R$.

This procedure for measuring the Casimir pressure $P\left(z_{i}\right)$ is very similar to our previous approach [11]. The main differences permitting us to perform measurements at practically the same separations $z_{i}(1 \leq i \leq 293)$ in each of $n=33$ sets of measurements are a $\sim 7 \%$ improvement in vibrational noise, and an improvement in the interferometric technique used to yield the distance $z_{\text {meas }}$ between the end of the fiber and the stationary reference. We implemented a two-color fiber interferometer similar to Ref. [17]. The use of this interferometer yielded an error $\Delta z_{\text {meas }}=0.2 \mathrm{~nm}$, and for every repetition of the Casimir pressure measurement we were able to reposition our sample to within $\Delta z_{\text {meas }}$.

The above experimental procedure permitted us to significantly suppress random errors, and made it unnecessary to resort to the cumbersome statistical approach to data processing used in Ref. [11]. The mean values of the Casimir pressure $\bar{P}\left(z_{i}\right)$ averaged over all 33 sets of measurements are shown as black crosses in Fig. 1(a)-1(f) in different separation subintervals. (Six subfigures are necessary to present all of the original experimental data with their respective error bars in true scales.) The horizontal arms of the crosses are equal to twice the absolute errors, $2 \Delta z=1.2 \mathrm{~nm}$, determined at $95 \%$ confidence in the measurement of separations between the zero roughness levels on the plate and on the sphere (see Ref. [11] for details). The vertical arms of the crosses are equal to twice the total absolute error, $2 \Delta P^{\operatorname{expt}}\left(z_{i}\right)$, determined at $95 \%$ confidence in the measurement of the Casimir pressure. The latter is a function of separation and is shown as the solid line in Fig. 2. $\Delta P^{\text {expt }}$ is the statistical combination of the random experimental error (shown as the long-dashed line in Fig. 2) obtained using the Student's $t$-distribution with $n-$ $1=32$ degrees of freedom, and of the systematic error (shown as the short-dashed line in Fig. 2). The systematic error in this experiment has two contributions: (i) the errors in the measurements of $\omega_{r}$ and $R$ [11], and (ii) the error from using the PFA. Note that in Refs. $[10,11]$ the latter was attributed to theory, whereas in our current approach the theoretical calculation of the Casimir pressure between two parallel plates is independent of the PFA. On the other hand, the equivalent experimental Casimir pressure in

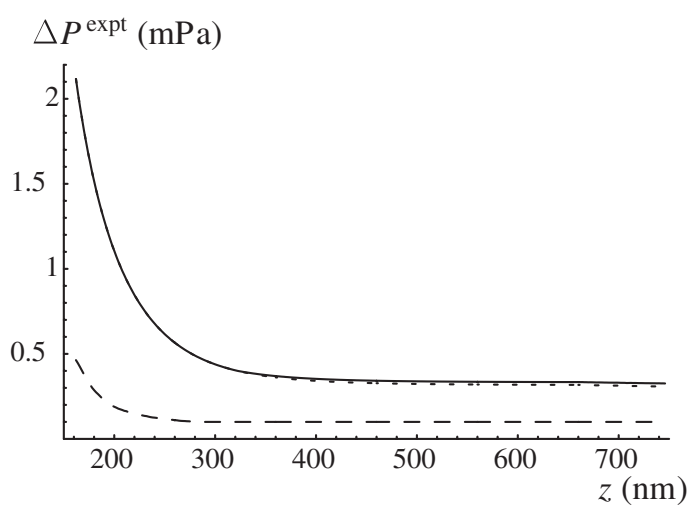

FIG. 2. The total absolute experimental error of the Casimir pressure measurements (solid line), random error (long-dashed line), and systematic error (short-dashed line) as functions of separation. 
Eq. (2) requires the error in the PFA to be attributed to the experimental systematic errors. As is seen in Fig. 2, it is the systematic error which now dominates the magnitude of the total experimental error $\Delta P^{\operatorname{expt}}\left(z_{i}\right)$. The dominance of the systematic error over the random error has never been previously achieved in Casimir force experiments. The total experimental relative error $\Delta P^{\operatorname{expt}} /\left|\bar{P}^{\operatorname{expt}}(z)\right|$ varies from $0.19 \%$ to $9.0 \%$ as the separation increases from 160 to $750 \mathrm{~nm}$. (The contribution from the use of the PFA to the total experimental error varies from $0.04 \%$ to $0.5 \%$, respectively.) At short separations our experimental precision has been improved by several times compared to our previous measurement [11].

To compare these experimentally determined pressures with theory, one needs to first characterize the electronic properties of the $\mathrm{Au}$ films used in the experiment. In previous experiments, these were obtained entirely from tabulated values. For a more conclusive comparison of experiment with theory we experimentally inferred the resistivity $\rho$ of the Au films as a function of temperature in the region from $T_{1}=3 \mathrm{~K}$ to $T_{2}=400 \mathrm{~K}$. Resistance measurements were performed on $\mathrm{Au}$ films deposited at the same time as the $\mathrm{Au}$ deposition on the oscillator, and the substrates were made of the same material as the Si plate of the oscillator. To do this a four probe approach on lithographically defined Au-strips was used. The resistivity of each sample was calculated by taking into account its geometrical factor (each strip was approximately $1 \mathrm{~mm}$ long, $10 \mu \mathrm{m}$ wide, and had the same thickness as the film deposited on the oscillator). The error on the resistivity of about $3 \%$ arises primarily from the difficulties in accurately measuring the sample's geometry. The resulting data for $\rho(T)$ at $T \gg T_{D} / 4$ (where $T_{D}=165 \mathrm{~K}$ is the Debye temperature for $\mathrm{Au}$ ) were fitted to a straight line. The slope of this line was then used to determine the magnitude of $\omega_{p}=8.9 \mathrm{eV}$. The value of $\gamma=0.0357 \mathrm{eV}$ at room temperature was determined from the smooth Drude extrapolation to lower frequencies [4] of the imaginary part of $\mathrm{Au}$ dielectric permittivity given by tabulated optical data [18]. Note that in previous work $[10,11]$ the values from tables, $\omega_{p}=9.0 \mathrm{eV}$ and $\gamma=0.035 \mathrm{eV}$, were used.

The experimental values of the Casimir pressure in Fig. 1 were compared with the Lifshitz theory [19] at room temperature $T=295 \mathrm{~K}$, using the calculation procedure presented in detail in Ref. [11]. Note that at nonzero Matsubara frequencies the use of the reflection coefficients expressed in terms of dielectric permittivity [12-14], or in terms of Leontovich impedance [15], leads to negligibly small differences in the final results. However, at zero frequency this is not the case. In terms of the Leontovich impedance, the reflection coefficients for the transverse magnetic (TM) and transverse electric (TE) modes are given by [15]

$$
r_{\mathrm{TM}}\left(0, k_{\perp}\right)=1, \quad r_{\mathrm{TE}}\left(0, k_{\perp}\right)=\frac{\omega_{p}-c k_{\perp}}{\omega_{p}+c k_{\perp}},
$$

where $k_{\perp}$ is the magnitude of the wave vector in the plane of the plates. In contrast, when the reflection coefficients are expressed in terms of the dielectric permittivity of the Drude model one finds [12,20]

$$
r_{\mathrm{TM}}\left(0, k_{\perp}\right)=1, \quad r_{\mathrm{TE}}\left(0, k_{\perp}\right)=0 .
$$

Importantly, Eq. (3) predicts small thermal effects at $z \leq$ $1 \mu \mathrm{m}$ in qualitative agreement with the case of ideal metals. However, Eq. (4) results in relatively large thermal effects up to $16 \%$ of the Casimir pressure at $z \leq 1 \mu \mathrm{m}$, and an $11 \%$ effect at $z=750 \mathrm{~nm}$. Further discussions on the applicability of Eqs. (3) and (4) can be found in Refs. [21,22].

The theoretical Casimir pressures were computed by using both Eqs. (3) and (4) (the Leontovich impedance approach and the Drude model approach, respectively). The surface roughness with variances equal to $3.6 \mathrm{~nm}$ on the plate and $1.9 \mathrm{~nm}$ on the sphere was taken into account by the geometrical averaging method [11]. Roughness contributes a correction of only $0.5 \%$ of the Casimir pressure at $z=160 \mathrm{~nm}$, and its contribution decreases with the increase of separation. The contributions of diffractiontype and correlation effects in the roughness correction, which are not considered in the geometrical averaging, were shown to be negligible [11]. The theoretical Casimir pressures taking into account the surface roughness are shown in Fig. 1(a)-1(f) by the light-gray bands (the Leontovich impedance approach), and by the darkgray bands (the Drude model approach). The width of the bands in the vertical direction is equal to twice the total theoretical error $2 \Delta P^{\text {theor }}(z)$, determined at $95 \%$ confidence. This error arises from the variation of the tabulated optical data and extrapolation parameters and results in a relative theoretical error $\Delta P^{\text {theor }}(z) /\left|P^{\text {theor }}(z)\right|$ equal to $0.5 \%$ [11]. Other factors, such as patch potentials or spatial nonlocality, were shown to be negligible (see Ref. [11] for more details). In our present approach to the comparison of experiment with theory, the theoretical pressures are not computed at the experimental points but rather within the measurement range from 160 to $750 \mathrm{~nm}$. Because of this, the error in the measurement of separation $\Delta z$ is irrelevant to theory and should be included in the analysis of experimental errors. As seen in Fig. 1(a)-1(f), the Leontovich impedance approach is consistent with data over the entire measurement range. We note that by using our new values of $\omega_{p}$ and $\gamma$, better agreement is achieved between the data and the impedance approach within the separation region from 200 to $400 \mathrm{~nm}$. With the values previously used, differences $\left(P^{\text {theor }}-P^{\text {expt}}\right)$ were changing nonmonotonically with decreasing $z$, but with the values determined here the expected monotonic dependence on $z$ is observed. We emphasize, however, that the variations in $P^{\text {theor }}$ using both sets of $\omega_{p}$ and $\gamma$ lie within the limit of theoretical error. In contrast, the Drude model approach, leading to relatively large thermal effects at $z \leq 1 \mu \mathrm{m}$, is excluded experimentally at $95 \%$ confidence over the entire measurement range from 160 to $750 \mathrm{~nm}$. If we choose a confidence level of $99 \%$ or $99.9 \%$, the Drude model approach is 


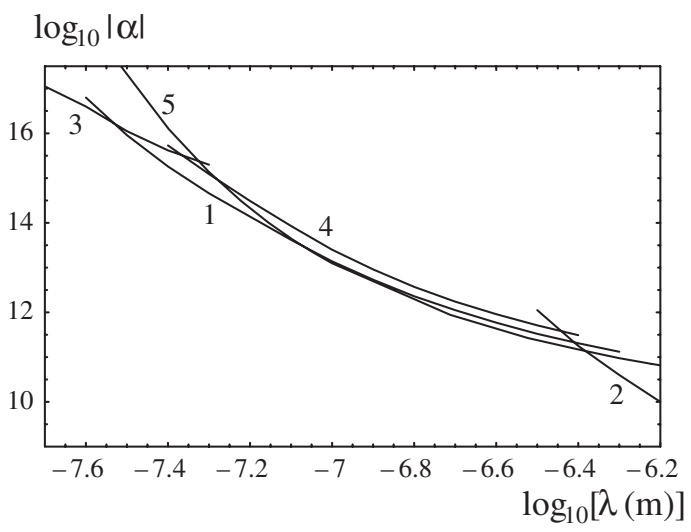

FIG. 3. Constraints on the parameters of the Yukawa interaction obtained in this paper (line 1), and from the experiments of Refs. [6,8,11,25] (lines 2, 3, 4, and 5, respectively). The region above each line is excluded, and that below is allowed.

excluded by the experimental data from 160 to $700 \mathrm{~nm}$, and from 210 to $620 \mathrm{~nm}$, respectively. The increase in the experimental precision achieved in this work, along with the use of more accurate values of $\omega_{p}$ and $\gamma$, allowed us to widen the interval in which the Drude model approach is excluded at 99\% confidence (in Ref. [11] from 300 to $500 \mathrm{~nm}$ ), and to demonstrate for the first time the exclusion of this model at the $99.9 \%$ confidence level. The Casimir pressure computed at $T=0$ is also consistent with our data. The small thermal effects predicted in Refs. [13-15] are currently experimentally inaccessible.

Our data can be used to impose stronger constraints on a Yukawa correction to the Newtonian gravitational potential predicted from the exchange of light hypothetical bosons
[23], and from extra-dimensional physics with a low unification scale [24]. The potential energy between two point masses $m_{1}$ and $m_{2}$ a distance $r$ apart arising from Newton's law with a Yukawa correction is given by

$$
V(r)=-G m_{1} m_{2}\left(1+\alpha e^{-r / \lambda}\right) / r
$$

where $G$ is the gravitational constant, $\alpha$ characterizes the strength of the Yukawa force, and $\lambda$ is its interaction range. The new constraints on $\alpha, \lambda$ are obtained using the same procedure as in Ref. [11], and are shown in Fig. 3 by line 1. In the same figure the constraints from earlier experiments are also shown. As seen in Fig. 3, the new constraints are the strongest in the region $29.5 \mathrm{~nm} \leq \lambda \leq 86 \mathrm{~nm}$ with the largest improvement by a factor of 3 at $\lambda \approx 40 \mathrm{~nm}$.

To summarize, our new results extend the range of separations in which we achieve a small experimental error. In addition, we have for the first time used the experimentally determined values of the electronic parameters of the samples when comparing to theory. This has permitted us to conclusively exclude the approach to the thermal Casimir force predicting large thermal effects at separations below $1 \mu \mathrm{m}$, and to strengthen constraints on non-Newtonian gravity. The experimental procedures we have developed will find applications in both fundamental physics and nanotechnology.

The work of E. F. was supported in part by DOE under the Contract No. DE-AC02-76ER071428. R. S. D. acknowledges NSF support through Grant No. CCF0508239. G. L. K. and V.M.M. were partially supported by DFG Grant No. 436 RUS 113/789/0-2.
[1] H. B. G. Casimir, Proc. K. Ned. Akad. Wet. 51, 793 (1948).

[2] V. M. Mostepanenko and N. N. Trunov, The Casimir Effect and Its Applications (Clarendon, Oxford, 1997); K. A. Milton, The Casimir Effect (World Scientific, Singapore, 2001).

[3] M. Kardar and R. Golestanian, Rev. Mod. Phys. 71, 1233 (1999).

[4] M. Bordag et al., Phys. Rep. 353, 1 (2001).

[5] S. K. Lamoreaux, Rep. Prog. Phys. 68, 201 (2005).

[6] S. K. Lamoreaux, Phys. Rev. Lett. 78, 5 (1997).

[7] U. Mohideen and A. Roy, Phys. Rev. Lett. 81, 4549 (1998); G.L. Klimchitskaya et al., Phys. Rev. A 60, 3487 (1999); G. Bressi et al., Phys. Rev. Lett. 88, 041804 (2002); F. Chen et al., Phys. Rev. Lett. 88, 101801 (2002); Phys. Rev. Lett. 97, 170402 (2006).

[8] B. W. Harris et al., Phys. Rev. A 62, 052109 (2000).

[9] H. B. Chan et al., Science 291, 1941 (2001).

[10] R. S. Decca et al., Phys. Rev. D 68, 116003 (2003).

[11] R. S. Decca et al., Ann. Phys. (N.Y.) 318, 37 (2005).
[12] M. Boström and B. E. Sernelius, Phys. Rev. Lett. 84, 4757 (2000).

[13] C. Genet et al., Phys. Rev. A 62, 012110 (2000).

[14] M. Bordag et al., Phys. Rev. Lett. 85, 503 (2000).

[15] B. Geyer et al., Phys. Rev. A 67, 062102 (2003).

[16] T. Emig et al., Phys. Rev. Lett. 96, 080403 (2006); D. E. Krause et al., Phys. Rev. Lett. 98, 050403 (2007).

[17] C. Yang et al., Opt. Lett. 27, 77 (2002).

[18] Handbook of Optical Constants of Solids, edited by E. D. Palik (Academic Press, New York, 1985).

[19] E. M. Lifshitz, Zh. Eksp. Teor. Fiz. 29, 94 (1956) [Sov. Phys. JETP 2, 73 (1956)].

[20] J. S. Høye et al., Phys. Rev. E 67, 056116 (2003).

[21] I. Brevik et al., Phys. Rev. E 71, 056101 (2005).

[22] V. B. Bezerra et al., Phys. Rev. E 73, 028101 (2006).

[23] E. Fischbach and C.L. Talmadge, The Search for NonNewtonian Gravity (Springer, New York, 1999).

[24] I. Antoniadis et al., Phys. Lett. B 436, 257 (1998).

[25] R. S. Decca et al., Phys. Rev. Lett. 94, 240401 (2005). 